\title{
La banalización del periodismo cultural. Show mediático y contenidos culturales en la prensa escrita peruana
}

Recibido: 16 de enero de 2017

Aceptado: 01 de agosto de 2017

Publicado: 27 de noviembre de 2017

\author{
Tomás Atarama-Rojas \\ tomas.atarama@udep.pe \\ Karold Rivera Ahumada \\ kar.riv.ahum@gmail.com \\ Universidad de Piura (Perú)
}

\begin{abstract}
Resumen: El periodismo cultural es uno de los campos informativos de mayor trascendencia en la vida social. Desde su nacimiento, ha estado ligado a la prensa escrita y al mundo de las ciencias, letras y artes, hasta convertirse hoy en un espacio de amplia y variada temática, que incluye no solo los acontecimientos más relevantes del mundo cultural, sino también los del entretenimiento y el espectáculo. Ante este escenario, esta investigación pretende demostrar, mediante un análisis de contenido de tres de los diarios nacionales más importantes de la prensa escrita peruana (El Comercio, La República, Perú21) que los contenidos culturales han sido reemplazados por cuestiones de farándula, chisme y hechos mediáticos, contribuyendo a una acepción cada vez más negativa del periodismo de espectáculos y a la banalización del verdadero periodismo cultural.
\end{abstract}

Palabras clave: Periodismo cultural, cultura, prensa escrita, periodismo de espectáculos, prensa peruana.

Abstract: Cultural Journalism is one of the most important fields of information in social life. Since its inception, it has been linked to the written press and to the world of sciences, arts and letters, and today, it has become a wide space of varied topics, which includes not only the most relevant events of the cultural world, but also those of entertainment and media show. Given this scenario, this research seeks to demonstrate, through a content analysis of three of the most important national newspapers in the peruvian written press (El Comercio, La República, Perú21) that cultural contents have been replaced by questions of "farandula", gossip and mediatic facts, contributing to an increasingly negative meaning of entertainment journalism and the trivialization of true Cultural Journalism. 
Key words: Cultural Journalism, Culture, Print Media, Entertainment Journalism, Peruvian Newspapers.

\section{Introducción}

La cultura es un elemento primordial del ejercicio periodístico. De hecho, el periodismo nació como un género cultural capaz de elevar el nivel de conocimiento del público, por lo que primaba su objetivo de formación antes que la información (Rodríguez, 2006). Es así como surge el periodismo cultural, entendido como un factor importante en la difusión y potenciación de la cultura, "ya sea a través de lecturas literarias o por medio de la contemplación de trabajos cinematográficos, o mediante su contextualización social, política, económica, etc. y la de sus creadores" (Arráez, Jensen y Pascual, 2014: 50).

Partiendo de su trascendencia en la vida y el desarrollo de la sociedad, es importante conocer cuál es o debería ser el rol de los profesionales que ejercen el periodismo cultural, pues no se trata de una profesión encargada de la simple transmisión de hechos generados alrededor del mundo cultural, de las artes o el entretenimiento; sino que "un buen periodismo cultural puede contribuir a digerir la información, a proponer piezas informativas o interpretativas que vayan más allá de una mera acumulación de datos en caída libre que abrumen a la ciudadanía" (Nieto, 2014: 133).

De esta manera, Bárcenas (2013: 17) sostiene que cualquier estudio, producto o contenido ligado a la cultura debe ser desarrollado teniendo en cuenta "las relaciones sociales y el sistema a través del cual se produce y se consume, y ese análisis de la cultura está íntimamente ligado con el estudio de la sociedad, la política y la economía”. Así también Jaakkola (2013) señala que, teniendo en cuenta el esfuerzo de los diarios por representar la vida cultural de su región y cubrir las cuestiones artísticas y culturales más relevantes, las páginas culturales de estos diarios deben reflejar los cambios generales en la posición y el papel de las artes en la sociedad.

A pesar de la importancia que enmarca la esencia del periodismo cultural para el hombre y la sociedad, en la actualidad, buena parte de la prensa escrita peruana no le otorga la valoración que este merece. Esta investigación pretende demostrar que, si bien la mayoría de los diarios nacionales cuenta con secciones de cultura y/o entretenimiento en sus ediciones impresas, los contenidos culturales publicados en estas han sido reemplazados por cuestiones de farándula, chisme y hechos mediáticos, contribuyendo a una acepción cada vez más negativa del periodismo de espectáculos y a la banalización del verdadero periodismo cultural.

Para comprobar esta hipótesis, ahondaremos, primero, en el concepto del periodismo cultural y su rol social. Luego, a través del análisis de contenido de tres diarios representativos de la prensa peruana, abordaremos tres grandes variables: $a$ ) la cobertura mediática de la información cultural en la prensa peruana, b) los temas de interés que priman en las secciones de cultura y c) la calidad periodística de los contenidos culturales en los diarios peruanos. Para tal estudio, hemos analizado 674 contenidos publicados en las secciones culturales de los tres diarios escogidos. 


\section{Periodismo cultural}

Si bien actualmente no existe una definición clara sobre el periodismo cultural, porque abarca un campo extenso y heterogéneo (Izquierdo, 2014; Hanush, 2015), es indiscutible la amplia atención que varios autores le han otorgado a su importancia o rol trascendental en la sociedad. De esta manera, Arráez, Jensen y Pascual (2014: 58) señalan que el periodismo cultural ejerce un papel relevante en la difusión y potenciación de la cultura en una determinada sociedad, puesto que "la enriquece mediante su desenvolvimiento no solo a nivel técnico o formal propio del manejo de los géneros periodísticos y de los distintos formatos, sino también en el plano del contenido del mensaje periodístico".

Asimismo, Izquierdo (2014) afirma que, teniendo en cuenta la especialización de los temas que abarca el periodismo cultural, ya sea pintura, literatura, cine, teatro o música, esta profesión contribuye a la difusión y divulgación del conocimiento, y ejerce una real influencia en la configuración de las ideas y el gusto del público de una época. El mismo autor señala que una correcta información cultural funciona como transmisora del patrimonio cultural y conlleva siempre aspectos educativos y pedagógicos que contribuyen con la formación de los lectores.

Por su parte, Delponti y Pestano (2012) analizan la influencia del periodismo cultural en la sociedad y sostienen que sus efectos se hacen evidentes con más facilidad que otros campos profesionales, pues (tanto por los contenidos que trabajan los periodistas de este campo, como por las formas en que presentan estos contenidos) algunas de las repercusiones de su ejercicio profesional recaen en la acentuación de las diferencias culturales de los públicos, los cuales son segmentados en grupos de elite, masa y popular.

Teniendo en cuenta la influencia y el rol social que radica en el periodismo cultural, autores como Rodríguez (2006) y Nieto (2014) exigen la necesidad de una rigurosa preparación por parte de los profesionales que buscan desempeñarse en este campo, así como una formación especializada por parte de los que ya ejercen esta profesión. Según Skulte (2015: 42), el periodista cultural tiende a comprender la información en todas sus dimensiones, "y se pregunta no sólo acerca de las razones, causas, objetivos y lugar del fenómeno en la vida social, sino también de algo tan variable como su significado y su nivel de importancia".

Se trata de una de las actividades profesionales que exigen una mayor preparación por parte de los periodistas que la ejercen, pues ellos "tienen el deber de proporcionar acceso al capital artístico a quienes no poseen los códigos, la formación intelectual y la sensibilidad necesarios para asimilarlo y convertirlo en gratificante" (Rodríguez, 2006: 10). Y es que al ser el periodismo cultural una especialidad importante del periodismo por el aporte social e intelectual que conlleva, no debemos dejar de lado la calidad de la información difundida, ya que (como en todo acto informativo) es el periodista el que se encarga (según su criterio personal) de determinar si el contenido trabajado es una información valiosa para el lector. De ahí que la exigente formación del profesional sea requisito indispensable para contribuir en la prudencia del informador a la hora de ejercer la actividad periodística, pues esta virtud le otorga los medios necesarios para elaborar una verdadera información (Atarama, 2012a). 
Así lo manifiesta Nieto (2014: 134), cuando sostiene que "el trabajo de calidad, la capacidad crítica, la formación, el manejo de las nuevas tecnologías e incluso el refuerzo de la marca profesional se hacen imprescindibles para garantizar la adaptación de los periodistas culturales a un contexto de extremada crudeza". Con esta afirmación, el mencionado autor hace referencia a la necesidad de una amplia y especializada formación de los profesionales del periodismo de cultura para alcanzar una labor profesional de calidad.

En este sentido, Arráez, Jensen y Pascual (2014) consideran que los periodistas especializados en cultura deben adecuar el mensaje del contenido al contexto del público al que se dirigen, deben ser capaces de asociar la información a los actuales fenómenos culturales y sociales que rodean a los lectores, no solo se trata de opinar o brindar una crítica sobre alguna actividad u obra cultural específica. Izquierdo (2014) afirma que una información cultural con un adecuado tratamiento periodístico cumple tres funciones: a) una función informativa, a través de noticias sobre acontecimientos culturales o educativos; $b$ ) una función mediadora, al generar una relación entre los artistas y el público, y c) una función orientadora, pues guía o ayuda al lector en la elección de determinadas propuestas culturales.

Ante la abundante información de hoy, la formación especial de los periodistas de cultura influye de manera positiva en los lectores, ya que los ayuda a discernir o distinguir obras de calidad frente a la carencia de ideas y la falta de complejidad de informaciones triviales. "La ciudadanía, abrumada por la sobrecarga informativa, necesita una mano experta, fiable, que cribe el aluvión de mensajes y extraiga el valor de las industrias culturales, y en este terreno el periodismo cultural tiene mucho que decir" (Nieto, 2014: 134).

\section{Cultura y espectáculos en la prensa escrita}

A lo largo de la historia de la prensa cultural, los términos "cultura" y "espectáculos" siempre han estado íntimamente relacionados. A principios del siglo XX, las informaciones relacionadas al espectáculo (ligado a todo tipo de manifestación y expresión artística humana) eran incluidas en las secciones o páginas de cultura de los diarios y revistas de la época. Este tipo de contenidos, referidos al cine, teatro, música y arte, fueron agrupadas bajo el nombre de "espectáculos", que también formaba parte del periodismo cultural. Sin embargo, el avance de las tecnologías y el auge del cine y la televisión de los noventas impulsaron una especie de separación entre el periodismo cultural y el periodismo de espectáculos (Ayala y León, 2000; Torres y Santos, 2014).

Este fenómeno se acentuó con la aparición de los diarios populares, la prensa amarillista y la creciente influencia del cine y la televisión, que dieron pase al llamado "info-entretenimiento", un nuevo espacio de la prensa, surgido por la necesidad de brindar al público información y entretenimiento. Rojas (2015: 50) define al infoentretenimiento como un género que implica no solo la elección de un determinado tipo de contenidos, sino también "todo un peculiar tratamiento de la información caracterizado por una presentación de los hechos más superficial y sensacionalista, el uso de un lenguaje coloquial y un estilo informal, la inclusión del rumor, la anécdota y el morbo del conflicto". 
Según Ayala y León (2000), otro de los factores que incide en la trivialización de los contenidos culturales, y en la separación del periodismo cultural y el de espectáculos es el desarrollo del llamado star system ("sistema de estrellas") o "farándula", un término que (en su sentido peyorativo) ha sido asociado con el mundo de las estrellas de la televisión y el cine, con la vida íntima de los actores, divas y personajes públicos, envueltos en un ámbito donde circulan los chismes y el rumor, y en el que el aumento de su fama es estimulada por las publicaciones de la prensa sensacionalista.

Es así como el significado de "espectáculo", antes ligado a la cultura y el arte, es reemplazado por el de "entretenimiento" y surgen términos como la "sociedad del espectáculo" o la "sociedad light", que hacen referencia a una sociedad pasiva en busca de un entretenimiento asociado al morbo, lujos y vanidades, adormecida por los contenidos superficiales de algunos medios informativos e industrias culturales que colocan el factor rentable por encima de productos de calidad (Vargas Llosa, 2012). Sobre esto también apunta algunas ideas Norgaard (2015), quien concibe al periodismo basado en el entretenimiento y los chismes de celebridades como una degradación de las artes y el periodismo cultural.

Ante este panorama, Arango (2015: 516) coincide y señala que "los críticos de la industria cultural denuncian permanentemente que lo bello, lo rimbombante y lo espectacular son los ganchos que utiliza la sociedad mediática para dejar que las audiencias se pierdan, masificadas, en el placer inmediato que proveen los medios". Asimismo, Rojas (2015: 52) sostiene que "desde que las noticias entraron a formar parte del mundo del espectáculo, muchos medios se han decantado hacia formatos de puro entretenimiento como base de su estrategia de negocio".

Al respecto, Atarama (2012b) señala que si bien algún dato o información puede generar interés en el público, el periodista debe analizar con criterio el contenido para determinar si contribuye, en alguna medida, al enriquecimiento del lector. De no ser así, el autor señala que el informador no puede legitimar un producto de baja calidad bajo el equivocado argumento de dar al público lo que desea para satisfacer su interés.

Como una solución ante el mal manejo de la información periodística y de entretenimiento, Galán (2014: 169) considera que "los nuevos medios de comunicación deberían distinguir claramente la información periodística del entretenimiento, tanto en los contenidos como en el aspecto industrial", aun cuando esta distinción o separación amenace el beneficio económico del medio en la actualidad.

\section{Materiales}

Partiendo de un breve repaso acerca del periodismo cultural y su relación con la prensa escrita, este estudio brinda un alcance de la actual situación de la prensa escrita peruana, respecto al desarrollo de contenidos culturales y de entretenimiento. El campo escogido para realizar esta investigación ha sido la prensa escrita, debido a que el surgimiento del periodismo cultural se da, precisamente, en este medio. Para el análisis de contenido, ana- 
lizamos 674 publicaciones de los diarios nacionales seleccionados para la muestra: $\mathrm{El} \mathrm{Co}$ mercio, La República y Perú21. Se ha tomado en cuenta la trayectoria, el nivel de lectoría y el tipo de contenidos periodísticos como criterios de selección.

El Comercio es uno de los diarios más antiguos del Perú. Su trayectoria y calidad profesional a lo largo de su historia, desde su fundación (1839) hasta la actualidad, lo han convertido en el primer diario de circulación nacional más prestigioso del país (Gestión, 2014). Posee una línea editorial de corte liberal, centroderecha y es el único diario peruano de prensa escrita que cuenta con revistas y suplementos de una amplia y variada temática: cultural, empresarial, económica, deportiva, hogarista, comercial y de entretenimiento. Uno de sus suplementos más importantes es "Luces", espacio asignado para las publicaciones diarias de cultura y entretenimiento.

La República es otro de los diarios de circulación nacional más antiguos de la prensa escrita peruana. Fue fundado en 1981 y, al igual que El Comercio (su principal competidor) se ha convertido en uno de los diarios de mayor prestigio en el país (La República, 2012). Tiene una línea editorial de corte centroizquierdista y forma parte del grupo Periódicos Asociados Latinoamericanos (PAL), al que pertenecen otras importantes casas editoriales de Latinoamérica (PAL, 2013). Este diario también cuenta con secciones variadas: "Política", "Opinión", "Economía", "Mundo", "Sociedad", "Deportes", "Ocio y Cultura" (sección cultural).

Perú 21 es un diario nacional que forma parte del grupo editorial El Comercio. Fue fundado en 2002 como un diario de corte político, dirigido a una determinada audiencia con intereses coyunturales de la política nacional (Gestión, 2014). Sin embargo, actualmente alberga tanto secciones de política, sociedad y cultura, como de entretenimiento o actualidad social. Su diseño vanguardista y la calidad de sus contenidos periodísticos lo han convertido, también, en uno de los diarios de mayor lectoría en la prensa peruana (CPI, 2017).

\section{Metodología}

Esta investigación se centra en un análisis de contenido de las secciones culturales de los diarios El Comercio, La República y Perú21 durante el mes de enero de 2017. Hemos seleccionado este periodo de tiempo debido a que consideramos que el primer mes del año representa uno de los de mayor actividad cultural en el país, pues, tanto para los jóvenes como para los adultos, es un periodo de vacaciones y ocio, y, por ende, de mayor asistencia a eventos culturales y de entretenimiento, de modo que es lógico pensar que la producción de noticias y/o contenidos culturales tiende a incrementarse en este tiempo.

El objetivo de este análisis es demostrar que los diarios impresos de circulación nacional han reemplazado los contenidos culturales por asuntos relacionados a la farándula, el chisme y los hechos mediáticos. Para comprobar esta hipótesis, se analizaron 674 publicaciones de las secciones de cultura y entretenimiento de los diarios que componen la muestra. En la figura 1 se observa el total de unidades analizadas, durante el mes de enero, por cada uno de los diarios en sus secciones de cultura. 
Figura 1: Publicaciones analizadas en la sección cultural de cada diario.

\begin{tabular}{|c|c|c|c|}
\hline DIARIO & $\begin{array}{c}\text { NOMBRE DE } \\
\text { LA SECCIÓN }\end{array}$ & FRECUENCIA DE PUBLICACIÓN & $\begin{array}{c}\text { TOTAL DE } \\
\text { PUBLICACIONES } \\
\text { CULTURALES } \\
\text { (ENERO 2017) }\end{array}$ \\
\hline El Comercio & $\begin{array}{c}\text { "Luces"/ } \\
\text { "Escape" }\end{array}$ & $\begin{array}{c}\text { "Luces"(lunes - miércoles y viernes - } \\
\text { domingo) / "Escape" (jueves) }\end{array}$ & 315 \\
\hline La República & "Ocio y Cultura" & Lunes - domingo & 169 \\
\hline Terú21 & "Escenarios" & Lunes - domingo & 190 \\
\hline TOTAL & & & $\mathbf{6 7 4}$ \\
\hline
\end{tabular}

Fuente: Elaboración propia.

Asimismo, en el desarrollo del análisis, se han considerado tres variables de estudio con sus respectivas categorías, que se detallan en los siguientes subapartados.

\subsection{Cobertura mediática de la información cultural}

El estudio de esta primera variable busca medir cuál es la real importancia que le dan los diarios peruanos a la información cultural. Para su estudio, además del total de publicaciones difundidas durante un mes por los diarios de la muestra, se toma en cuenta el género periodístico usado para cada información publicada en la sección cultural y/o de entretenimiento de los diarios, ya sea de carácter informativo, interpretativo o de opinión.

En el primero se analiza el contenido, según noticia o entrevista; en el segundo se clasifica la información en crónica o reportaje; y en el tercero se analiza si es columna, comentario, crítica o reseña. También se examina el tipo de publicación, según el espacio asignado por los diarios para cada una de estas, teniendo en cuenta la ubicación y la extensión de las mismas.

De esta forma, clasificaremos cada información entre seis tipos: especial (cuando la nota o el contenido ocupa más de una página), principal (cuando ocupa una página completa), abridora (cuando la información abre la página y ocupa media carilla), secundaria (cuando se ubica a un margen y ocupa la cuarta parte de una carilla o abarca menos columnas que la nota abridora), breve (cuando abarca solo un párrafo) y complementaria (cuando forma parte de otra publicación o se ubica dentro de ella).

\subsection{Temas de interés en las secciones de cultura}

Tomando como referencia el objeto de información de cada publicación cultural difundida por los tres diarios escogidos, en la segunda variable se analizarán los temas que más des- 
tacan en la sección cultural de estos medios. De esta manera, según el hecho noticioso o el tema central recogido por las publicaciones, clasificaremos los contenidos en tres grupos:

- Temas vinculados al espectáculo, cuando el tema central de las publicaciones sea la farándula (información sobre la vida íntima de personajes mediáticos) o los escándalos mediáticos.

- Temas vinculados a la industria cultural y/o entretenimiento, cuando el hecho noticioso de los contenidos esté relacionado a la televisión, el cine o la música.

- Temas vinculados al arte y la cultura tradicional, cuando se trate de publicaciones sobre festividades y/o tradiciones (información ligada a la historia y/o costumbres de un determinado pueblo o cultura), literatura, teatro o artes visuales (toda la información vinculada a la fotografía, escultura, pintura o cualquier tipo de artes plásticas).

A través de esta clasificación, podremos comprobar si las secciones culturales de los diarios nacionales están directamente relacionadas con la publicación de contenidos sobre cultura popular o tradicional, o si, más bien, están más orientadas a la difusión de temas del espectáculo como la farándula y los escándalos mediáticos.

\subsection{Calidad periodística de los contenidos}

En la tercera y última variable, se analizarán aspectos periodísticos de los contenidos publicados en las secciones de cultura y/o entretenimiento de los tres diarios seleccionados, teniendo en cuenta la calidad de la información. Para esta parte del análisis, se examina, primero, el lenguaje empleado en cada uno de los contenidos (técnico, formal o coloquial), así como las fuentes utilizadas para legitimar los datos y la información. De esta forma, en cada publicación analizaremos si las fuentes citadas son directas, secundarias, indirectas o no oficiales.

Si bien podemos incluir a las fuentes secundarias dentro de las indirectas, en este caso, hemos creído conveniente dividirlas teniendo en cuenta que los diarios suelen recoger testimonios o declaraciones (incluso de los mismos protagonistas de la noticia) hechas en otros medios de comunicación para justificar la credibilidad de su información, por lo que, lejos de ser fuentes secundarias, son testimonios o declaraciones obtenidos de otros medios y entrarían al grupo de fuentes indirectas. Para el caso de las fuentes no oficiales, hemos incluido dentro de este rango las notas que contienen rumores o datos no confirmados, así como las publicaciones que no exponen o citan alguna fuente.

Por último, en esta variable también analizaremos la relevancia de la información difundida. Para ello, clasificaremos cada contenido de la sección cultural de los diarios en hecho mediático (cuando el hecho o tema central del contenido sea puramente mediático) o hecho de relevancia informativa (cuando, además de saciar el puro interés de los lectores, la información contribuya o influya en la vida de estos).

Es importante resaltar que hemos clasificado como hecho mediático a todos los contenidos que tienen como tema central algún acontecimiento escandaloso, polémico o visible sólo 
por la popularidad de sus protagonistas o que buscan, únicamente, saciar el interés de los lectores, sin ánimo de contribuir en algún aspecto de la vida de estos. Mientras que dentro de hecho de relevancia informativa, hemos incluido las publicaciones que destacan temas netamente culturales, con aportes que, además de abordar el interés de los lectores, contribuyen en su formación con contenidos de utilidad.

Considerando estas tres variables de estudio (con sus respectivas categorías e indicadores) se ha diseñado un cuadro para el análisis de cada información publicada en la sección cultural o de entretenimiento de los tres diarios nacionales que conforman la muestra. A continuación, se presenta la figura 2 para el análisis de contenido.

Figura 2: Tabla de análisis de contenido.

\begin{tabular}{|c|c|c|}
\hline VARIABLE & CATEGORÍA & INDICADOR \\
\hline \multirow[b]{2}{*}{$\begin{array}{l}\text { COBERTURA } \\
\text { MEDIÁTICA }\end{array}$} & Género periodístico & $\begin{array}{l}\text { - Informativo: noticia, entrevista. } \\
\text { - Interpretativo: reportaje, crónica. } \\
\text { - De opinión: columna, comentario, crítica, reseña. }\end{array}$ \\
\hline & $\begin{array}{l}\text { Tipo de publicación } \\
\text { según espacio } \\
\text { asignado }\end{array}$ & $\begin{array}{l}\text { - Especial. } \\
\text { - Principal. } \\
\text { - Abridora. } \\
\text { - Secundaria. } \\
\text { - Breve. } \\
\text { - Complementaria. }\end{array}$ \\
\hline \multirow{3}{*}{$\begin{array}{l}\text { TEMAS DE INTERÉS EN LAS } \\
\text { SECCIONES DE CULTURA }\end{array}$} & $\begin{array}{l}\text { Temas vinculados al } \\
\text { espectáculo }\end{array}$ & $\begin{array}{l}\text { - Farándula. } \\
\text { - Escándalos mediáticos. }\end{array}$ \\
\hline & $\begin{array}{l}\text { Temas vinculados a la } \\
\text { industria cultural y/o } \\
\text { entretenimiento }\end{array}$ & $\begin{array}{l}\text { - Televisión. } \\
\text { - Cine. } \\
\text { - Música. }\end{array}$ \\
\hline & $\begin{array}{l}\text { Temas vinculados } \\
\text { al arte y la cultura } \\
\text { tradicional }\end{array}$ & $\begin{array}{l}\text { - Festividades / tradiciones. } \\
\text { - Literatura. } \\
\text { - Teatro. } \\
\text { - Artes visuales. }\end{array}$ \\
\hline \multirow{3}{*}{$\begin{array}{l}\text { CALIDAD PERIODÍSTICA } \\
\text { DEL CONTENIDO }\end{array}$} & Lenguaje & $\begin{array}{l}\text { - Técnico. } \\
\text { - Formal. } \\
\text { - Coloquial. }\end{array}$ \\
\hline & Fuentes & $\begin{array}{l}\text { - Directas. } \\
\text { - Secundarias. } \\
\text { - Indirectas. } \\
\text { - No oficiales. }\end{array}$ \\
\hline & $\begin{array}{l}\text { Relevancia de la } \\
\text { información }\end{array}$ & $\begin{array}{l}\text { - Hecho mediático. } \\
\text { - Hecho de relevancia informativa. }\end{array}$ \\
\hline
\end{tabular}

Fuente: Elaboración propia. 


\section{Resultados y reflexiones}

Para el estudio de cada variable, hemos agrupado los resultados de la investigación es seis tablas, teniendo en cuenta el análisis y la comparación de resultados de cada una de las categorías, tanto a nivel individual (de cada diario) como a nivel general (de los tres diarios en conjunto). Asimismo, además del análisis de los resultados, anotamos algunas reflexiones que nos pueden ayudar a comprender la problemática central que aborda esta investigación, así como la importancia del estudio en general.

\subsection{Cobertura mediática de la información cultural}

Según los resultados, casi la mitad (40.1\%) de las publicaciones culturales de los principales medios peruanos son secundarias, es decir, ocupan un margen de la página del diario o simplemente menos columnas que la noticia principal o abridora. El diario que hace un mayor uso de este tipo de publicaciones es Perú 21 , ya que el 18.3\% de contenidos secundarios analizados pertenecen a su sección cultural (figura 3 ).

Figura 3: Cobertura mediática de la información según el espacio asignado.

\begin{tabular}{|c|c|c|c|c|c|c|c|}
\hline $\begin{array}{c}\text { ESPACIO } \\
\text { ASIGNADO }\end{array}$ & ESPECIAL & PRINCIPAL & ABRIDORA & SECUNDARIA & BREVE & COMPLEMENTARIA & \\
\hline El Comercio & $8.8 \%$ & $3.4 \%$ & $18.5 \%$ & $12.3 \%$ & $3.7 \%$ & $0.1 \%$ & $46.8 \%$ \\
\hline La República & $0.1 \%$ & $4.0 \%$ & $4.3 \%$ & $9.5 \%$ & $7.1 \%$ & $0.0 \%$ & $25.0 \%$ \\
\hline Perú21 & $0.1 \%$ & $0.1 \%$ & $7.3 \%$ & $18.3 \%$ & $2.4 \%$ & $0.0 \%$ & $28.2 \%$ \\
\hline $\begin{array}{c}\text { TOTAL } \\
\text { GENERAL }\end{array}$ & $\mathbf{9 . 0} \%$ & $\mathbf{7 . 5} \%$ & $\mathbf{3 0 . 1} \%$ & $\mathbf{4 0 . 1} \%$ & $\mathbf{1 3 . 2} \%$ & $\mathbf{0 . 1} \%$ & $\mathbf{1 0 0 . 0} \%$ \\
\hline
\end{tabular}

Fuente: Elaboración propia.

Otra de las publicaciones que destaca en los diarios, según los resultados, es la abridora. Como muestra la figura 3, del 30\% de publicaciones de este tipo, el $18.5 \%$ pertenece a $E l$ Comercio. Y es que, teniendo en cuenta el estudio realizado, podemos decir que una de las razones de este último resultado es el espacio que ofrece este diario para la sección cultural, pues, a diferencia de los otros (La República y Perú21), asigna un suplemento especial ("Luces") para las publicaciones culturales. Este hecho también justifica el alto porcentaje que muestra El Comercio respecto a las publicaciones especiales, que ocupan más de una página del diario. Según la tabla de porcentajes, los contenidos especiales de El Comercio abarcan casi la totalidad (8.8\%) de las publicaciones especiales de los tres diarios. Podemos decir que, prácticamente, es el único diario que difunde notas especiales de cultura.

De los resultados obtenidos en este primer apartado, podemos deducir que los diarios impresos peruanos no le otorgan un espacio amplio a los contenidos de carácter cultural, pues, 
como hemos visto, la mayoría de sus publicaciones culturales son secundarias y abridoras. Y es que, como podremos observar más adelante, esto se debe al género periodístico usado con mayor frecuencia en la sección cultural de los diarios, en la que escasean los géneros interpretativos que, por profundidad del contenido, implican el uso de un espacio más extenso. Asimismo, es importante resaltar que, mientras que El Comercio otorga un suplemento especial de 16 páginas para los contenidos culturales, La República y Perú21 solo tienen una sección cultural que abarca de una a dos páginas.

El género periodístico que destaca en la sección cultural de los diarios analizados es el informativo, pues el $87 \%$ de sus publicaciones culturales son noticias y entrevistas. Como se observa en la figura 4 , en los tres diarios sobresale la noticia como subgénero principal.

Figura 4: Cobertura mediática de la información según el género periodístico.

\begin{tabular}{|c|c|c|c|c|c|c|c|c|c|}
\hline \multirow{2}{*}{$\begin{array}{c}\text { GÉNERO } \\
\text { PERIODÍSTICO }\end{array}$} & \multicolumn{2}{|c|}{$\begin{array}{c}\text { GÉNEROS } \\
\text { INFORMATIVOS }\end{array}$} & \multicolumn{2}{c|}{$\begin{array}{c}\text { GÉNEROS } \\
\text { INTERPRETATIVOS }\end{array}$} & \multicolumn{3}{c|}{ GÉNEROS ARGUMENTATIVOS/DE OPINIÓN } \\
\cline { 2 - 10 } & Noticia & Entrevista & Crónica & Reportaje & Columna & Comentario & Crítica & Reseña & \\
\hline El Comercio & $32.3 \%$ & $2.1 \%$ & $0.6 \%$ & $5.5 \%$ & $0.7 \%$ & $0.7 \%$ & $1.3 \%$ & $3.4 \%$ & $46.7 \%$ \\
\hline La República & $20.8 \%$ & $3.6 \%$ & $0.0 \%$ & $0.0 \%$ & $0.0 \%$ & $0.1 \%$ & $0.0 \%$ & $0.6 \%$ & $25.1 \%$ \\
\hline Perú21 & $26.1 \%$ & $2.1 \%$ & $0.0 \%$ & $0.0 \%$ & $0.0 \%$ & $0.0 \%$ & $0.0 \%$ & $0.0 \%$ & $28.2 \%$ \\
\hline $\begin{array}{c}\text { TOTAL } \\
\text { GENERAL }\end{array}$ & $79.2 \%$ & $7.7 \%$ & $0.6 \%$ & $5.5 \%$ & $0.7 \%$ & $0.9 \%$ & $1.3 \%$ & $4.0 \%$ & $100.0 \%$ \\
\hline
\end{tabular}

Fuente: Elaboración propia.

Asimismo, es importante destacar que El Comercio, a diferencia de La República y Perú21, utiliza también el género interpretativo y de opinión en sus publicaciones culturales, por lo que, como hemos podido observar en el desarrollo del estudio, el suplemento asignado a sus contenidos culturales ("Luces") no solo muestra noticias, sino también reportajes especiales y reseñas sobre aspectos culturales relacionados a las artes y letras. Sin embargo, el porcentaje de estos dos géneros sigue siendo mínimo (6.1\%) en comparación al $34.4 \%$ que representa el género informativo en la información cultural de este diario (figura 4).

La ausencia de los géneros interpretativos y argumentativos es más notoria en las secciones culturales de La República y Perú21, en las que (como se aprecia en la figura 4) la totalidad de sus publicaciones culturales pertenecen al género informativo. Teniendo en cuenta el análisis realizado, podemos decir que la ausencia de estos géneros se debe a que estos dos diarios no apuestan por la profundidad de temas o contenidos culturales que puedan aportar datos relevantes y dar lugar a la interpretación o la crítica. 


\subsection{Temas de interés}

Solo a excepción de El Comercio, la farándula es el tema que más destaca en la sección cultural de los diarios peruanos. Como se observa en la figura 5, el $26 \%$ de los contenidos culturales de los diarios tiene como tema central la farándula, en los que (según hemos podido comprobar en el desarrollo del análisis) se muestran diversos asuntos de la vida privada de personajes mediáticos o artistas famosos como hecho central de la publicación.

Sin embargo, a diferencia de La República y Perú21, el tema que predomina en las publicaciones de El Comercio es la televisión, que abarca el mayor porcentaje (11.5\%) de todos los temas que aparecen en sus contenidos culturales. Esto, sin duda, obedece a la frecuencia de publicaciones que difunde este diario sobre estrenos de series y programas de televisión de medios extranjeros y peruanos, según hemos podido ver en el análisis realizado. De hecho, son los temas vinculados a la industria cultural y entretenimiento los que predominan en el espacio cultural de El Comercio. Según los resultados de la figura 5, el 22.5\% de sus contenidos están relacionados a la televisión, el cine o la música.

Figura 5: Tema central de la información cultural.

\begin{tabular}{|c|c|c|c|c|c|c|c|c|c|c|}
\hline \multirow{2}{*}{$\begin{array}{c}\text { TEMA } \\
\text { CENTRAL DEL } \\
\text { CONTENIDO }\end{array}$} & \multicolumn{2}{|c|}{$\begin{array}{c}\text { TEMAS VINCULADOS } \\
\text { AL ESPECTÁCULO }\end{array}$} & \multicolumn{2}{|c|}{$\begin{array}{c}\text { TEMAS VINCULADOS } \\
\text { A LA INDUSTRIA } \\
\text { CULTURAL Y/O } \\
\text { ENTRETENIMIENTO }\end{array}$} & \multicolumn{3}{|c|}{$\begin{array}{c}\text { TEMAS VINCULADOS AL ARTE Y LA CULTURA } \\
\text { TRADICIONAL }\end{array}$} \\
\cline { 2 - 13 } & Farándula & $\begin{array}{c}\text { Escándalos } \\
\text { mediáticos }\end{array}$ & TV & Cine & Música & $\begin{array}{c}\text { Festividades } \\
/ \text { tradiciones }\end{array}$ & Literatura & Teatro & $\begin{array}{c}\text { Arte } \\
\text { Visual }\end{array}$ \\
\hline El Comercio & $68 \%$ & $0.4 \%$ & $11.5 \%$ & $4.5 \%$ & $6.5 \%$ & $0.3 \%$ & $3.4 \%$ & $4.9 \%$ & $8.5 \%$ & $46.8 \%$ \\
\hline La República & $7.3 \%$ & $3.4 \%$ & $2.8 \%$ & $5.3 \%$ & $3.6 \%$ & $0.4 \%$ & $0.6 \%$ & $1.3 \%$ & $0.3 \%$ & $25.0 \%$ \\
\hline Perú21 & $12.0 \%$ & $5.8 \%$ & $3.0 \%$ & $3.7 \%$ & $3.6 \%$ & $0.0 \%$ & $0.1 \%$ & $0.0 \%$ & $0.0 \%$ & $28.2 \%$ \\
\hline $\begin{array}{c}\text { TOTAL } \\
\text { GENERAL }\end{array}$ & $26.1 \%$ & $9.6 \%$ & $17.3 \%$ & $13.5 \%$ & $13.7 \%$ & $0.7 \%$ & $4.1 \%$ & $6.2 \%$ & $8.8 \%$ & $100.0 \%$ \\
\hline
\end{tabular}

Fuente: Elaboración propia.

A pesar de que la farándula es el tema que más destaca en las publicaciones de los diarios (sobre todo en Perú21 y La República) a nivel general, podemos decir que los temas favoritos de la sección cultural de los diarios nacionales son los relacionados a la industria cultural y/o entretenimiento. Según los resultados, el $44.5 \%$ de los contenidos resaltan asuntos relacionados a estos temas, pues gran parte de las publicaciones corresponden a noticias sobre conciertos musicales y estrenos de películas. No obstante, la prevalencia de estos temas también obedece a la cantidad de publicaciones que difunde el diario $E l$ Comercio, pues, como lo mencionamos anteriormente, casi la mitad de estos contenidos $(22.5 \%)$ pertenece solo a este diario.

Es importante resaltar que (al igual que la televisión) el cine y la música, la farándula y los escándalos mediáticos son otros de los temas predominantes de las secciones culturales de los diarios peruanos, pues el $35.7 \%$ de las publicaciones tienen como tema central asuntos 
relacionados al espectáculo, sobre todo a la farándula, como ya lo hemos mencionamos en el primer párrafo de este apartado. El diario que difunde más publicaciones vinculadas a la farándula y los escándalos mediáticos es Perú21, ya que la mitad de los contenidos de esta categoría (17.8\%) pertenece solo a este diario (figura 5).

No podemos dejar de mencionar que la difusión de contenidos referidos al arte y la cultura tradicional es casi inexistente en las secciones culturales de los diarios nacionales. De hecho, casi la totalidad de contenidos que resaltan estos temas pertenecen solo a El Comercio (17.1\%), mientras que en La República y Perú21, la difusión de estos contenidos no llega ni al 3\% (figura 5), lo que hace aún más notoria la primacía de la farándula y la ausencia de los temas netamente culturales (como la literatura o las artes visuales) en la sección cultural de los diarios.

\subsection{Calidad periodística de los contenidos}

Como se muestra en la figura 6 , los principales diarios peruanos utilizan el lenguaje formal en los contenidos de su sección cultural. El $85.3 \%$ de sus publicaciones son redactadas con un lenguaje formal, que obedece al género periodístico utilizado para cada uno de sus contenidos.

Figura 6: Lenguaje usado en los contenidos culturales de los diarios peruanos.

\begin{tabular}{|c|c|c|c|c|}
\hline $\begin{array}{c}\text { LENGUAJE } \\
\text { UTILIZADO }\end{array}$ & TÉCNICO & FORMAL & COLOQUIAL & \\
\hline El Comercio & $6.4 \%$ & $40.1 \%$ & $0.3 \%$ & $46.7 \%$ \\
\hline La República & $0.1 \%$ & $21.7 \%$ & $3.3 \%$ & $25.1 \%$ \\
\hline Perú21 & $0.0 \%$ & $23.6 \%$ & $4.6 \%$ & $28.2 \%$ \\
\hline TOTAL GENERAL & $6.5 \%$ & $85.3 \%$ & $8.2 \%$ & $100.0 \%$ \\
\hline
\end{tabular}

Fuente: Elaboración propia.

No obstante, al alto empleo del lenguaje formal por los principales diarios nacionales, $\mathrm{El}$ Comercio (a diferencia de La República y Perú21) sí utiliza en un mínimo porcentaje (6.4\%) el lenguaje técnico en algunos de sus contenidos culturales. Esto es entendible, teniendo en cuenta que El Comercio es el único diario que hace uso de géneros interpretativos y argumentativos en su sección cultural, por lo que el empleo del lenguaje técnico se evidencia, en algunas ocasiones, en sus reportajes, reseñas o críticas vinculadas a las artes visuales.

Como se observa en la figura 7 , los principales diarios nacionales utilizan fuentes indirectas para atribuir la información de sus publicaciones culturales. El $60.8 \%$ de los contenidos analizados en su sección cultural muestra este tipo de fuentes. De acuerdo con este estudio, de estas fuentes, las que más destacan son las declaraciones obtenidas de revistas internacionales, programas de televisión y redes sociales de los sujetos protagonistas de la noticia. 
Figura 7: Fuentes usadas en las publicaciones culturales de los diarios peruanos.

\begin{tabular}{|c|c|c|c|c|c|}
\hline $\begin{array}{c}\text { TIPO DE } \\
\text { FUENTES }\end{array}$ & DIRECTAS & SECUNDARIAS & INDIRECTAS & $\begin{array}{c}\text { NO } \\
\text { OFICIALES }\end{array}$ & \\
\hline El Comercio & $10.1 \%$ & $0.1 \%$ & $34.1 \%$ & $2.4 \%$ & $46.7 \%$ \\
\hline La República & $7.6 \%$ & $0.9 \%$ & $10.4 \%$ & $6.2 \%$ & $25.1 \%$ \\
\hline Perú21 & $5.5 \%$ & $1.2 \%$ & $16.3 \%$ & $5.2 \%$ & $28.2 \%$ \\
\hline $\begin{array}{c}\text { TOTAL } \\
\text { GENERAL }\end{array}$ & $23.1 \%$ & $2.2 \%$ & $60.8 \%$ & $13.8 \%$ & $100.0 \%$ \\
\hline
\end{tabular}

Fuente: Elaboración propia.

Por otro lado, solo un $23 \%$ de las publicaciones culturales de los diarios nacionales contienen fuentes directas. El 10\% de este tipo de fuentes pertenece a los contenidos de El Comercio. Volviendo al análisis realizado, es importante reflexionar aquí que quizá el número de periodistas asignados a la sección cultural de los diarios sea una de las razones por las que El Comercio haga un mayor uso de fuentes directas. Y es que, si bien en la mayoría de los contenidos culturales no aparece el autor de las notas (pues la gran mayoría son noticias con fuentes recogidas de otros medios) en El Comercio, contrario a lo que sucede en La República y Perú21, sí aparece el autor del contenido en varias de sus publicaciones, sobre todo cuando se trata de entrevistas, reportajes, reseñas o críticas relacionadas a temas de exposiciones, escultura o literatura.

De este modo, podemos decir que (a excepción de El Comercio) los diarios nacionales no cuentan con un determinado grupo de periodistas encargado exclusivamente de la investigación, el manejo de datos y fuentes directas para el desarrollo de las publicaciones de su sección cultural.

Los hechos de relevancia informativa tratados en las publicaciones culturales de los principales diarios peruanos solo superan en $1.4 \%$ a los hechos puramente mediáticos que difunden estos medios en su sección cultural. Como muestra la figura 8 , el 50.7\% de las publicaciones culturales de los diarios tratan hechos de relevancia informativa. Sin embargo, El Comercio posee más de la mitad de este porcentaje (35.2\%).

Figura 8: Relevancia de la información según el hecho principal de la publicación.

\begin{tabular}{|c|c|c|c|}
\hline $\begin{array}{c}\text { TIPO DE HECHO } \\
\text { PRINCIPAL }\end{array}$ & HECHO MEDIÁTICO & $\begin{array}{c}\text { HECHO DE } \\
\text { RELEVANCIA } \\
\text { INFORMATIVA }\end{array}$ & TOTAL \\
\hline El Comercio & $11.6 \%$ & $35.2 \%$ & $46.7 \%$ \\
\hline La República & $15.7 \%$ & $9.3 \%$ & $25.1 \%$ \\
\hline Perú21 & $22.0 \%$ & $6.2 \%$ & $28.2 \%$ \\
\hline TOTAL GENERAL & $49.3 \%$ & $50.7 \%$ & $100.0 \%$ \\
\hline
\end{tabular}

Fuente: Elaboración propia. 
Asimismo, como se puede apreciar en la figura 9, El Comercio es el único diario en el que los hechos de relevancia informativa superan en más del $20 \%$ a la publicación de hechos mediáticos. Por el contrario, La República y Perú21 muestran un mayor porcentaje de hechos mediáticos en sus contenidos culturales. La diferencia es mayor en el caso del diario Perú21, en el que los hechos mediáticos superan en más del 15\% a los hechos de relevancia informativa.

Figura 9: Relevancia de la información según el hecho principal de la publicación.

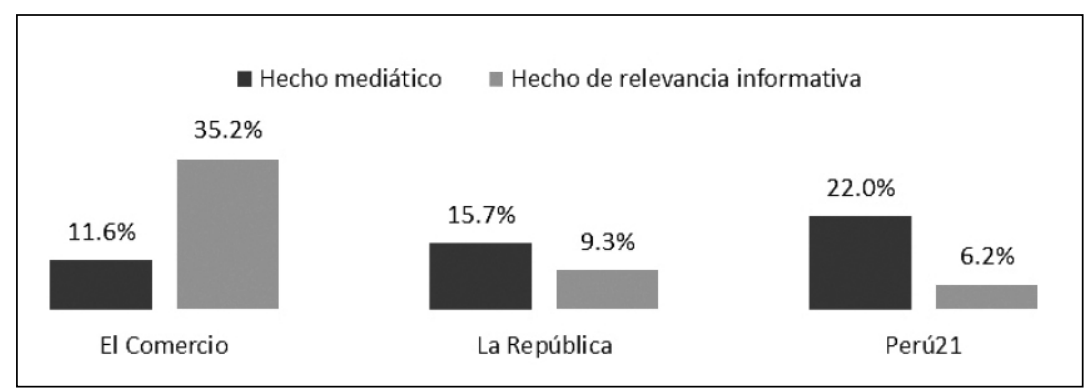

Fuente: Elaboración propia.

Este contraste entre los porcentajes parciales de los diarios analizados y el resultado global del estudio en esta categoría responde al hecho de que la mayor parte de publicaciones culturales que difunden los diarios peruanos (46.7\%) pertenece solo a El Comercio. De este modo, los resultados parciales de este diario, respecto a los hechos de relevancia informativa, se reflejan también en las cifras globales del estudio.

Es necesario resaltar que esta última categoría está muy vinculada con la variable de los temas de interés, analizada anteriormente. De hecho, una de las principales razones que explican los resultados en esta categoría es que (como lo mencionamos anteriormente) la farándula es el tema que más destaca en la sección cultural de los diarios peruanos (figura $5)$, por lo que es probable que el porcentaje de hechos mediáticos $(49.3 \%)$ esté relacionado en gran parte a grandes eventos o noticias sobre la vida privada de personajes mediáticos sin ninguna interpretación o aporte cultural por parte del autor. Así lo demuestran las cifras en la figura 4, en la que se aprecia que (a excepción de El Comercio) los diarios nacionales no utilizan los géneros interpretativos o argumentativos en sus publicaciones culturales. De hecho, los temas culturales como las artes visuales o la literatura solo son trabajados con profundidad por El Comercio a través de reportajes, comentarios o reseñas.

\section{Conclusiones}

Teniendo en cuenta el análisis realizado en esta investigación, podemos afirmar que si bien la prensa escrita peruana difunde contenidos de relevancia informativa en sus secciones de cultura y/o entretenimiento, casi la mitad de sus publicaciones $(49.3 \%)$ pertenecen a hechos puramente mediáticos, relacionados a temas de farándula y televisión, en los que, 
más allá de brindar una información valiosa que contribuya a enriquecer la vida y el conocimiento del lector (como rol fundamental del periodismo cultural) solo se limita a saciar el interés morboso y comercial del público: el primero, al divulgar la cotidianidad y la vida privada de personajes mediáticos y artistas famosos; y el segundo, al difundir simple información sobre las producciones audiovisuales más exitosas del momento, sin ningún análisis o interpretación de sus contenidos que permita a los lectores discernir entre productos mediocres o de calidad.

Sin embargo, es importante destacar que (a pesar de que en La República y Perú21 los hechos mediáticos y los temas de farándula superan a los hechos de verdadera relevancia informativa y a los temas netamente culturales) El Comercio sí maneja una notable cobertura de la información cultural en su edición impresa. De hecho, de las 674 publicaciones analizadas, durante un mes, en la sección cultural de tres diarios peruanos, 315 pertenecen a El Comercio. Como lo hemos señalado en el análisis de algunas categorías, este diario (a diferencia de los otros) asigna un suplemento especial exclusivo para sus contenidos culturales. Por ende, podemos decir que es el único diario peruano que otorga un papel fundamental al periodismo cultural.

Por otra parte, los resultados en el análisis de la cobertura mediática y la relevancia de la información cultural que muestra este estudio nos permite afirmar el papel trivial o secundario que cumple el periodismo cultural en la prensa escrita peruana. A pesar de que todos los diarios cuentan con una sección especial para las publicaciones de cultura y/o entretenimiento, la profundidad y la extensión de sus contenidos es superficial, es decir, están más ligados a un "periodismo light", como afirma Vargas Llosa (2012), que al criterio trascendental que enmarca el verdadero periodismo cultural en la sociedad. Es así que, en cuanto al espacio asignado, el mayor porcentaje de publicaciones (40\%) de los diarios son de carácter secundario. Esto significa que son noticias de poca extensión, con escaso desarrollo y profundidad.

Asimismo, el género periodístico y las fuentes usadas en los contenidos revelan la escasa rigurosidad con la que se maneja la información cultural en la prensa escrita peruana. Así lo demuestra el ínfimo uso de los géneros interpretativos y argumentativos en los contenidos culturales de los diarios, en los que, del 13\% de las publicaciones que usan estos géneros, el $12.3 \%$ pertenece a El Comercio.

Otro de los indicadores que muestra el trabajo superficial en la información cultural de los diarios es el alto porcentaje de fuentes indirectas que se encuentran en los contenidos $(60.8 \%)$, que, lejos de legitimar la calidad de los datos y la información de las publicaciones, denotan la carente investigación por parte de los periodistas en temas culturales: según el análisis realizado en esta investigación, la mayoría de las fuentes utilizadas (tanto en Perú21 como en La República) provienen de otros canales informativos o de las cuentas de redes sociales de los sujetos protagonistas de la información; por lo que, muchas veces, lo que se muestra en los contenidos son declaraciones de personajes sacadas de otros medios. De hecho, en algunas ocasiones, las publicaciones de los diarios llegan a ser casi iguales por el uso de los mismos datos y fuentes. 
Después de haber realizado este estudio cuantitativo sobre la cobertura del periodismo cultural en los principales diarios peruanos, hemos podido demostrar que si bien los diarios impresos de circulación nacional difunden información sobre temas culturales y/o de entretenimiento, en la mayoría de ellos (La República y Perú21) los contenidos mediáticos referidos a la farándula y la industria cultural (televisión, cine y música) superan a los hechos netamente culturales de relevancia informativa, lo que termina en la difusión de refritos o productos de baja calidad, no sólo por la temática cultural, sino también por la poca profundidad y rigurosidad periodística con la que se trata este tipo de información.

No obstante, a lo mencionado anteriormente, es importante diferenciar la labor del diario El Comercio, que si bien coincide en muchos indicadores con los demás diarios, el manejo y la calidad de sus contenidos culturales están más ligados al verdadero periodismo cultural antes que a lo mediático, al menos así lo demuestra en su edición impresa.

\title{
Fuentes consultadas
}

Arango, C. (2015). "Industrias culturales y estética. Un rastreo sobre su posible relación". Palabra Clave. Vol. XVIII, núm. 2, pp. 499-536. Extraída el 22/III/2016 desde https://goo. $\mathrm{gl} /$ LcWULy

Arráez, R.; Jensen, E. y Pascual, C. (2014). "Periodismo cultural en la postmodernidad". Historia y Comunicación Social, vol. XIX, pp. 49-61. Extraída el 18/X/2017 desde https:// revistas.ucm.es/index.php/HICS/article/download/45107/42476

\begin{abstract}
Atarama, T.
_(2012a). "La prudencia en la inmediatez del acto informativo". Ecos de la Comunicación, núm. 5, pp. 95-11. Extraída el 21/III/2016 desde https://goo.gl/r6mVtT

_(2012b). "El reconocimiento de la persona como requisito para el ejercicio prudente de la actividad informativa”. Comhumanitas. Vol. III, núm. 1, pp. 23-36. Extraída el 18/X/2017 desde https://goo.gl/TWbkop
\end{abstract}

Ayala, K. y León, E. (2000). El periodismo cultural y el de espectáculos. Trayectoria en la prensa escrita. Lima siglos XIX y XX. Lima: USMP.

Bárcenas, C. (2013). “Aproximaciones al estudio de la convergencia cultural”. Estudios sobre las culturas contemporáneas, núm. 38, pp. 9-27. Extraída el 18/X/2017 desde http:// www.redalyc.org/html/316/31629858002/

CPI, Compañía Peruana de Estudios de Mercado y Opinión Pública (2017). "Estudio de lectoría de diarios en lima y 15 principales ciudades - 2016". Extraída el 17/X/2017 desde https://goo.gl/rfPTJC 
Delponti, P. y Pestano, J. (2012). "El papel del periodismo cultural en la construcción simbólica de un imaginario social globalizado". Actas IV Congreso Internacional Latina de Comunicación Social: Comunicación, control y resistencias. Extraída el 17/X/2017 desde https://goo.gl/tmkMbV

Galán, J. (2014). "Cuando el 'cuarto poder' se constituye en cuarto poder: propuestas". Palabra Clave. Vol. XVII, núm. 1, pp. 152-187. Extraída el 17/X/2017 desde http://goo. $\mathrm{gl} / \mathrm{p} 05 \mathrm{xZS}$

Gestión (2014). “El Comercio conmemora los 175 años de su fundación”. Extraída el 26/X/2017 desde https://goo.gl/oeBGGw

Hanush, F. (2015). "Cultural Forces in Journalism: The impact of cultural values on Māori journalists' professional views”. Journalism Studies, Vol. XVI, núm. 2, pp. 191-206. Extraída el 18/X/2017 desde http://goo.gl/RiNyPq

Izquierdo, V. (2014). “Análisis de la información artística en los medios escritos generalistas españoles (El País, El Mundo y ABC)”. Estudios sobre el Mensaje Periodístico. Vol. XX, pp. 1121-1130. Extraída el 18/X/2017 desde https://goo.gl/71PGCo

Jaakkola, M. (2013). "Diversity through Dualism. The Balancing Principle as an Organizational Strategy in Culture Departments of Newspapers". Nordicom Review, núm. 34, pp. 89-98. Extraída el 18/X/2017 desde http://goo.gl/vIFjB0

La República (2012). "La República, un diario que apuesta por la lectura”. Extraída el 18/X/2017 desde https://goo.gl/5ASvXf

Nieto, M. (2014). "Periodismo cultural y concentración de medios. La infoxicación en la difusión de las industrias culturales y su estado en las redes sociales”. Extraída el 18/X/2017 desde https://goo.gl/gQJAEr

Norgaard, N. (2015). Cultural Journalism and Cultural Critique in a changing Media Landscape. Journalism Practice, Vol. IX, 760-772. Recuperado el 04 de abril de 2016, de http://goo.gl/vS7NTe.

PAL, Periódicos Asociados Latinoamericanos (2013). "20 casas editoriales, de 12 países, con los periódicos de mayor prestigio en todas sus plataformas". Extraída el 18/X/2017 desde https://goo.gl/x8LtmP

Rodríguez, F. (2006). Periodismo cultural. Madrid: Síntesis.

Rojas, J. (2015). "La creciente banalización de los contenidos deportivos". Cuadernos de periodistas: revista de la Asociación de la Prensa de Madrid, núm. 31, pp. 48-56. Extraída el 18/III/2016 desde https://goo.gl/ZZizcB 
Skulte, I. (2015). "The Concept of Cultural Journalism: What the editors in Latvia think they do when doing Cultural Journalism". Journalism Research, núm. 08, pp. 38-69. Extraída el 4/IV/2016 desde http://goo.gl/JLfTMW

Torres, M. y Santos, D. (2014). "Trends and transformations within cultural journalism: a case study of newsmagazine Visão”. Observatorio. Vol. VIII, núm. 4, pp. 171-185. Extraída el 18/X/2017 desde http://goo.gl/YIa8Xd

Vargas Llosa, M. (2012). La civilización del espectáculo. Madrid: Alfaguara. 